\title{
Exchange-correlation errors at harmonic and anharmonic orders: the case of bulk $\mathrm{Cu}$
}

\author{
SHOBHANA NARASIMHAN* and STEFANO DE GIRONCOLI ${ }^{\dagger}$ \\ Theoretical Sciences Unit, Jawaharlal Nehru Centre for Advanced Scientific Research, Jakkur PO, \\ Bangalore 560 064, India \\ ${ }^{\dagger}$ Scuola Internazionale Superiore di Studi Avanzati and Istituto Nazionale per la Fisica della Materia Via Beirut 2-4, \\ I-34014 Trieste, Italy
}

\begin{abstract}
As an aid towards improving the treatment of exchange and correlation effects in electronic structure calculations, it is desirable to have a clear picture of the errors introduced by currently popular approximate exchange-correlation functionals. We have performed ab initio density functional theory and density functional perturbation theory calculations to investigate the thermal properties of bulk $\mathrm{Cu}$, using both the local density approximation (LDA) and the generalized gradient approximation (GGA). Thermal effects are treated within the quasiharmonic approximation. We find that the LDA and GGA errors for anharmonic quantities are an order of magnitude smaller than for harmonic quantities; we argue that this might be a general feature. We also obtain much closer agreement with experiment than earlier, more approximate calculations.
\end{abstract}

Keywords. Anharmonicity; density functional theory; exchange; correlation; copper.

\section{Introduction}

The development of density functional theory (Hohenberg and Kohn 1964; Kohn and Sham 1965) has revolutionized computational condensed matter physics, making it possible to compute most properties of solids $a b$ initio, with no empirical input apart from the atomic numbers of elements. In the process of mapping the many-electron Schrödinger equation onto a one-electron equation, an exchange-correlation (XC) term is introduced into the one-electron Hamiltonian, which contains all the many body effects. The main problem in the field of $a b$ initio electronic structure calculations is that we do not know this XC potential exactly. Though the two most commonly used approximations, the local density approximation (LDA) and the generalized gradient approximation (GGA) work well for many systems, they do introduce errors, which become particularly serious for strongly correlated systems. Understanding and improving the approximations for the XC potentials is one of the main goals in the field of electronic structure calculations today.

In the LDA, the XC term is approximated by the $\mathrm{XC}$ potential for a homogeneous electron gas of the local density. It is well known that the LDA tends to overbind, giving lattice constants that are too small, and cohesive energies that are too high. The GGA includes terms involving the gradient of the local density, and therefore might be expected to work better; however, experience has shown that this is not always the case. Frequently, the

\footnotetext{
*Author for correspondence
}

GGA appears to overcorrect for the LDA errors, giving an underbinding. Systematic studies (Favot and Dal Corso 1999) have shown that these trends are also manifested in harmonic properties, with the LDA giving phonon frequencies and bulk moduli that are too high (compared to experiment), and the GGA giving values that are too low. In our work, we wish to go beyond this earlier work on static and harmonic properties, and examine what happens for anharmonic properties.

The anharmonic terms in the Hamiltonian (third and higher order powers in a Taylor-series expansion of the Hamiltonian in powers of atomic displacements away from equilibrium) lead to the lattice constant, bulk modulus and phonon frequencies changing as a function of temperature and/or pressure. We will compute these effects for copper, for which experimental data has been available for decades. While there are some previous calculations on the thermal behaviour of copper (MacDonald and MacDonald 1981; Moruzzi et al 1988; Caðin et al 1999), these have all involved additional approximations either about the form of the interatomic potentials or about the treatment of thermal effects. The agreement between these earlier calculations and experimental data is not particularly good, and we would also like to see whether it is possible to do a better job, within the limitations imposed by the approximate nature of the $\mathrm{XC}$ potentials used.

\section{Ab initio calculations}

We have performed density functional theory calculations using the packages PWSCF and PHONON (Baroni 
et al 2001). The nuclear potential has been described by an ultrasoft pseudopotential (Vanderbilt 1990), and the Kohn-Sham equations are expanded in a plane wave basis set with a cut-off of $30 \mathrm{Ry}$ (increased to $300 \mathrm{Ry}$ for the expansion of the augmentation charges introduced by the use of the ultrasoft pseudopotential). Total energies and phonon dynamical matrices are evaluated using a grid of $60 \mathrm{k}$ points in the irreducible Brillouin zone. To deal with possible convergence problems, we use both the Methfessel-Paxton smearing scheme (Methfessel and Paxton 1989) and smearing using the Fermi-Dirac occupation factor corresponding to the temperature of interest. Phonon dynamical matrices are evaluated using density functional perturbation theory (Baroni et al 1987) on a $4 \times 4 \times 4$ grid in the first Brillouin zone; the dynamical matrices for a $20 \times 20 \times 20$ grid are then obtained by Fourier interpolation. Two different XC potentials are used: for the LDA, the parametrization by Perdew and Zunger (1981); and for the GGA, the Perdew-BurkeErnzerhof form (Perdew et al 1996).

\section{Results}

At zero temperature, we find the total energy for a range of lattice constants, $a$, using both LDA and GGA. Upon fitting these results to the fourth-order Birch-Murnaghan equation of state (Birch 1947), our results for the static lattice constant, $a_{0}$, the bulk modulus, $B_{0}$, and the pressure derivative of the bulk modulus, $B_{0}{ }^{\prime}$, are 6.71 bohr, 1.72 MBar and 5.0 respectively when using the LDA; the corresponding GGA results are 6.94 bohr, 1.28 MBar and $5 \cdot 11$, respectively. The experimental values (at room temperature) of $a_{0}$ and $B_{0}$ are $6.82 \mathrm{bohr}$ and 1.37 , respectively (Kittel 1996); there is a wide scatter in the experimentally reported values for $B_{0}{ }^{\prime}$. These results are consistent with the tendency mentioned above, that the LDA overbinds and the GGA underbinds. One striking feature of these results is that while the LDA and GGA values for the harmonic quantity, $B_{0}$, differ by the rather large amount of $29 \%$, the LDA and GGA results for the anharmonic quantity, $B_{0}{ }^{\prime}$, differ only by $2 \%$.

Using the values obtained for the static lattice constant, we have evaluated the phonon frequencies, $\omega_{2 \lambda}$ (which are harmonic quantities) and the mode Grüneisen parameters, $\gamma_{q} \lambda$ (the corresponding anharmonic quantities, describing how the phonon frequencies vary with volume), along high-symmetry directions of the Brillouin zone. These results are plotted in figure 1, along with experimental results for the phonon frequencies (Drexel 1972; Nilsson and Rolandson 1973; Lynn et al 1973). Once again, we notice that: (i) the experimental values lie sandwiched between LDA and GGA values and (ii) the discrepancy between LDA and GGA values for the (harmonic) phonon frequencies is significantly larger than that between LDA and GGA values for the (anharmonic)
Grüneisen parameters. Averaged over the Brillouin zone, the discrepancy in the phonon frequencies is $11.6 \%$, which is significantly higher than the discrepancy in the Grüneisen parameters of $1.5 \%$.

In addition to examining how quantities change with the application of pressure, one can also examine how properties vary as a function of temperature. When analysing the effects of $\mathrm{XC}$ potential at harmonic and higher orders, it should be noted that quantities such as the coefficient of thermal expansion depend upon both harmonic and anharmonic terms in the Hamiltonian. To incorporate the effects of finite temperature, we will make use of the quasiharmonic approximation for the free energy of the crystal at temperature, $T$,

$$
F(a, T)=E_{\text {stat }}(a)+k_{\mathrm{B}} T \sum_{\mathbf{q} \lambda} \ln \left\{2 \sin h\left(\frac{\hbar \omega_{\mathbf{q} \lambda}(a)}{2 k_{\mathrm{B}} T}\right)\right\},
$$

where $E_{\text {stat }}(a)$ is the static energy at lattice constant $a$, and the second term involves summing over all phonon modes (with wavevector, $\mathbf{q}$ and polarization, $\lambda$ ), the vibrational free energy of a single harmonic oscillator of frequency, $\omega_{\lambda \lambda} ; k_{\mathrm{B}}$ and $\hbar$ are Boltzmann's constant and Planck's constant, respectively. There is no explicit anharmonicity in this quasiharmonic expression; however, the anharmonicity is implicitly included by allowing the phonon frequencies to depend on the lattice constant, $a$, and we use density functional perturbation theory to compute $\omega_{\lambda \lambda}(a)$ for a range of lattice constants.

At each temperature, $T$, the curve obtained using (1) for $F(a, T)$ is fit to the fourth-order Birch-Murnaghan

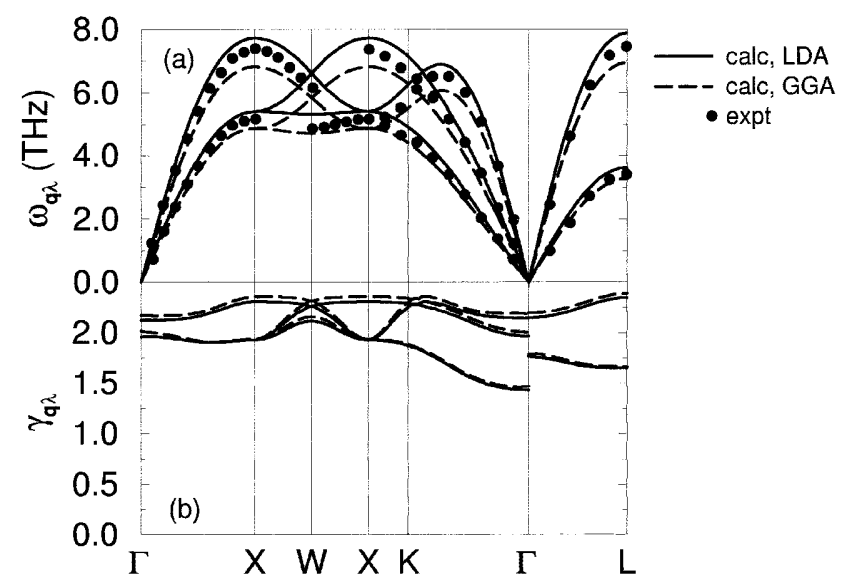

Figure 1. Dispersion along high-symmetry directions of the Brillouin zone of (a) phonon frequencies, $\omega_{\mathrm{q} \lambda}$ and (b) mode Grüneisen parameters, $\gamma_{\mathbf{q} \lambda}$, evaluated at the static lattice constant. The solid and dashed lines are the results obtained using LDA and GGA, respectively, the dots are experimental points at room temperature (Drexel 1972; Nilsson and Rolandson 1973; Lynn et al 1973). Note that the discrepancy between LDA and GGA results is much less in (b) than in (a). 
equation of state so as to obtain $a_{0}, B_{0}$ and $B_{0}{ }^{\prime}$ as a function of temperature. Figures 2(a) and (b) show our results for $B_{0}$ and $\partial B_{0} / \partial T$ as a function of temperature, along with the experimental results (Chang and Hultgren 1965). From these figures, it is clear that, yet again, while both the LDA and GGA make relatively large (and opposite) errors in the harmonic quantity, $B_{0}$, the discrepancy between LDA and GGA results and the error relative to experiment are significantly reduced upon looking instead at the anharmonic quantity, $\partial B_{0} / \partial T$.

Finally, in figure 3, we have plotted our results for the coefficient of linear expansion $\alpha$ as a function of temperature, obtained by differentiating our results for the lattice constant as a function of temperature. It is seen from the figure that the LDA underestimates not only the static lattice constant (as mentioned above), but also the coefficient of thermal expansion at all temperatures; therefore, the LDA error in the lattice constant increases with temperature. The same is true for the GGA error in the lattice constant, though in this case $a$ and $\alpha$ are overestimated, not underestimated. It is important to note that the error in $\alpha$ can be traced almost entirely to errors made at harmonic order, since $\alpha=\gamma C_{V} / 3 B_{0}$, where $C_{V}$ is the specific heat capacity at constant volume, and $\gamma$ is a weighted average of the Grüneisen parameters evaluated over the entire Brillouin zone. Especially at temperatures above the Debye temperature, where all modes are excited

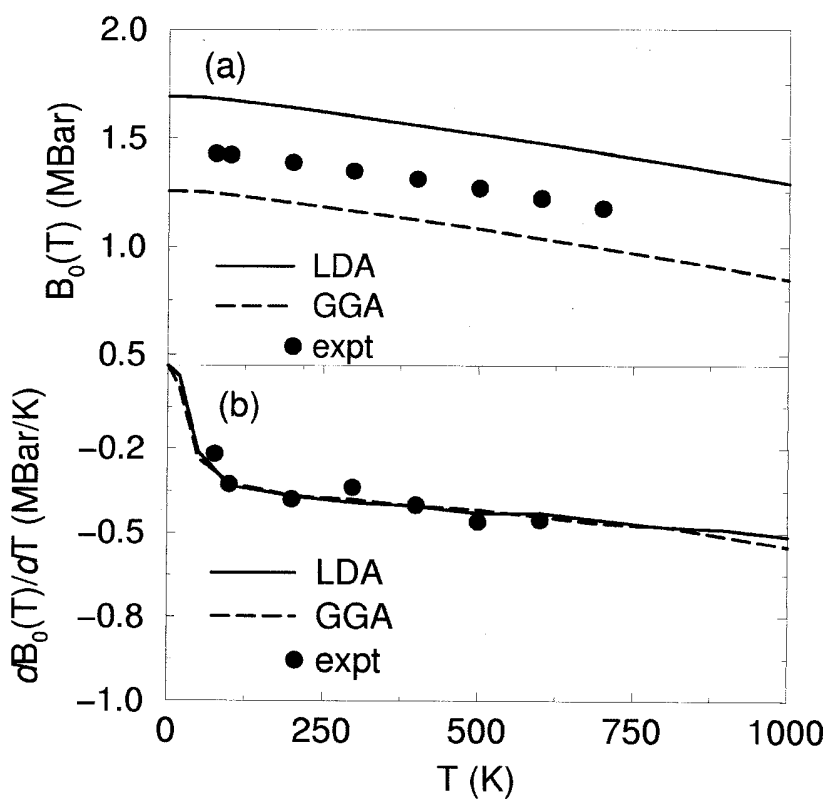

Figure 2. Variation with temperature, $T$ of (a) the bulk modulus, $B_{0}$ and (b) $\partial B_{0} / \partial T$, the rate of change of the bulk modulus with temperature. The solid and dashed lines are the results obtained using LDA and GGA, respectively, the dots are experimental points (Chang and Hultgren 1965). Note that though LDA and GGA make relatively large errors in $B_{0}(T)$, $\partial B_{0} / \partial T$ is obtained very accurately. and $C_{V}$ has reached its maximum value of $3 k_{\mathrm{B}}$, the errors in $\alpha$ must arise from errors in $\gamma$ and/or $B_{0}$. While the former error is negligible, it can be seen from figure 2(a) that the latter error is fairly large. By evaluating this expression, we have verified that the error in $\alpha$ is almost entirely due to the large error in $B_{0}$. We have also checked that the errors made in $C_{V}$ are negligible at lower temperatures (Narasimhan and de Gironcoli 2002).

\section{Discussion}

From the results given in the previous section, one very clear trend that is immediately obvious is that the errors due to using an approximate $\mathrm{XC}$ functional (LDA or GGA) are larger by about an order of magnitude for harmonic properties than for the corresponding anharmonic properties. This is an important result that does not appear to have been pointed out before for any system. However, in retrospect, this result can be understood quite simply, if one assumes that the root cause of the LDA and GGA errors in static, harmonic and anharmonic properties is the incorrect value obtained for the lattice constant. Evaluating the various quantities considered in this paper corresponds to computing various derivatives of the energy with respect to atomic coordinates, and the errors in these quantities arise from our having evaluated these derivatives at the wrong equilibrium positions. However, if the energy is expanded as a polynomial in powers of atomic coordinates, the dependence on lattice constant of the $n$th derivative becomes progressively smaller as $n$ increases. Thus, the error in harmonic quantities (arising from evaluating the second derivative at the wrong lattice constant) can be expected to be larger than

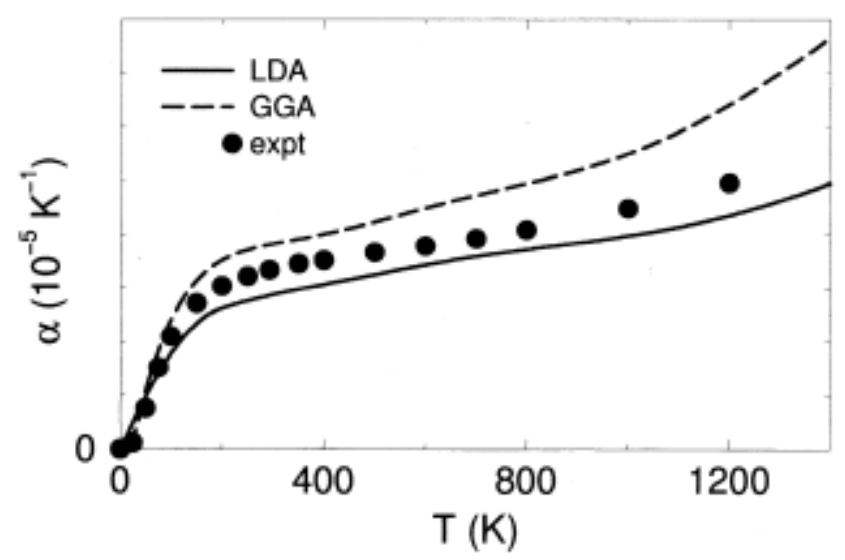

Figure 3. Coefficient of linear expansion, $\alpha$, as a function of temperature, $T$. The solid and dashed lines are the results obtained using LDA and GGA, respectively, the dots are experimental points (from AIP Handbook of Physics 1973). Note that LDA and GGA make opposite errors in $\alpha$, and that these errors increase with $T$. 
the error in anharmonic quantities (arising from evaluating third and higher order derivatives at the wrong lattice constant).

Another conclusion that can be drawn from the results in the previous section is that the error in the lattice constant will increase with temperature, for both LDA and GGA; this could be important to note when performing, for example, $a b$ initio molecular dynamics simulations at high temperatures.

Though we have performed calculations for just one element, copper, we have reason to believe that these features (much smaller errors in anharmonic than in harmonic quantities, and an increase in the LDA and GGA errors with temperature) might hold for all materials, due to the generality of the arguments cited above. However, we plan to carry out calculations on other materials to confirm whether this is indeed the case.

In this paper we have focussed mainly on the errors made due to the approximate nature of the $\mathrm{XC}$ potential used. However, it should be pointed out that the agreement between our results and experimental data is still quite good, and is in fact better than that obtained in earlier calculations in which either an empirical model potential was used (MacDonald and MacDonald 1981; Caðin et al 1999) and/or thermal effects were treated in an approximate way (MacDonald and MacDonald 1981; Moruzzi et al 1988).

\section{Summary}

We have investigated the performance of the local density approximation and the generalized gradient approximation in predicting the thermal properties of copper $a b$ initio, using the quasiharmonic approximation for the free energy. We find that the LDA and GGA errors in anharmonic quantities are smaller by an order of magnitude than the errors in harmonic quantities; this can be explained by simple arguments. We also obtain much better agreement with experiment than earlier more approximate calculations.

\section{Acknowledgements}

(SN) thanks the Associateship program of the AbdusSalam International Centre for Theoretical Physics, Trieste, Italy, which made this collaboration possible. (SdG) has been supported in part by MURST under COFIN99, and by the Iniziativa Trasversale Calcolo Parallelo of INFM.

\section{References}

Baroni S, Giannozzi P and Testa A 1987 Phys. Rev. Lett. 58 1861

Baroni S, Dal Corso A, de Gironcoli S and Giannozzi P 2001 http://www.pwscf.org

Birch F 1947 Phys. Rev. 71809

Caðin T, Dereli G, Uludoðan M and Tomak M 1999 Phys. Rev. B59 3468

Chang Y A and Hultgren R 1965 J. Phys. Chem. 694162

Drexel W 1972 Z. Phys. 255281

Favot F and Dal Corso A 1999 Phys. Rev. B60 11427

Hohenberg P and Kohn W 1964 Phys. Rev. B136 864

Kittel C 1996 Introduction to solid state physics (New York: John Wiley \& Sons) 7 th ed

Kohn W and Sham L J 1965 Phys. Rev. A140 1133

Lynn J W, Smith H G and Nicklow R M 1973 Phys. Rev. B8 3493

MacDonald R A and MacDonald W M 1981 Phys. Rev. B24 1715

Methfessel M and Paxton A T 1989 Phys. Rev. B40 3616

Moruzzi V L, Janak J F and Schwarz K 1988 Phys. Rev. B37 790

Narasimhan S and de Gironcoli S 2002 Phys. Rev. B65 064302

Nilsson G and Rolandson S 1973 Phys. Rev. B7 2393

Perdew J P and Zunger A 1981 Phys. Rev. B23 5048

Perdew J P, Burke K and Ernzerhof M 1996 Phys. Rev. Lett. 77 3865

Vanderbilt D 1990 Phys. Rev. B41 7892 